\title{
Commentary: Fontan survivor-outwit, outlast, outplay but do not overstay (your welcome)
}

\author{
Kenneth E. Mah, MD, MS, a,b and David S. Cooper, MD, MPH ${ }^{\mathrm{a}, \mathrm{b}}$
}

From ${ }^{\mathrm{a} D e p a r t m e n t}$ of Pediatrics, University of Cincinnati College of Medicine, Cincinnati, Ohio; and ${ }^{\mathrm{b}}$ The Heart Institute, Division of Cardiology, Cincinnati Children's Hospital Medical Center, Cincinnati, Ohio. Disclosures: Authors have nothing to disclose with regard to commercial support.

Received for publication Nov 28, 2018; accepted for publication Nov 29, 2018; available ahead of print Jan 23, 2019.

Address for reprints: Kenneth E. Mah, MD, MS, The Heart Institute, Cincinnati Children's Hospital Medical Center, 3333 Burnet Ave, MLC 2003, Cincinnati, OH 45229 (E-mail: Kenneth.Mah@ @chmc.org).

J Thorac Cardiovasc Surg 2019;157:2014-5

$0022-5223 / \$ 36.00$

Copyright $₫ 2018$ by The American Association for Thoracic Surgery

https://doi.org/10.1016/j.jtcvs.2018.11.118

Cardiac care teams have long sought factors that impact both short- and long-term outcomes of patients with univentricular physiology who undergo the Fontan procedure. ${ }^{1-4}$ The surgical advances in performing the Fontan procedure, including staged palliation and its modification (ie, fenestration), have further complicated our quest for understanding. In this issue of the Journal, Ono and colleagues ${ }^{5}$ approach this question by focusing on intensive care unit length of stay (ICU-LOS), factors associated with ICU-LOS, and the relationship between ICU-LOS and late outcomes. The study suggests that longer ICU-LOS was associated with preoperative factors (anatomic factors [hypoplastic left heart syndrome, anomalous systemic venous drainage] and elevated preoperative transpulmonary gradient), operative factors (concomitant atrioventricular valve procedure, cardiopulmonary bypass [CPB] time, and surgical era) and postoperative factors (lower aortic saturation, pleural effusion, chylothorax, ascites, and infection). Furthermore, ICU-LOS was found to be associated with late mortality and cardiac reoperation.

Although these findings are of interest, there are various aspects of the analysis and its interpretation thereof that warrant reflection and consideration. Preoperative, intraoperative, and postoperative analyses did not include variables previously associated with ICU-LOS, such as postoperative ventricular function, acute kidney injury (AKI), and fluid

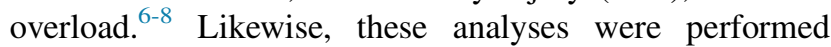
separately, thus, not controlling for confounders, and the impact may be overstated, given the focus on univariate analyses and phrasing that implies causation. The late outcomes analysis is also difficult to interpret because it includes both ICU-LOS and the factors the study associated with ICU-LOS while omitting other variables (ie, heterotaxy syndrome). There were additional issues with variable selection, such as exclusion of 30-day mortality patients in ICU-LOS calculations, lack of a definition for "longer ICU-LOS," and lack of definition/baseline for low arterial

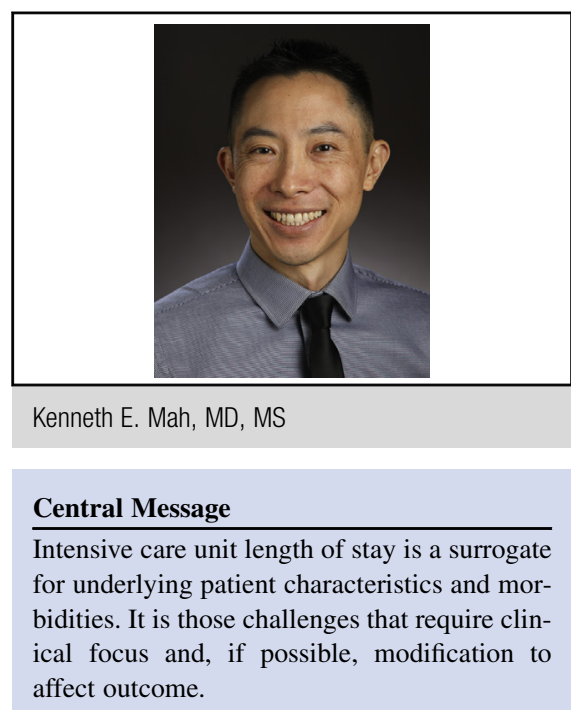

See Article page 2005.

oxygen saturation. Furthermore, it is unclear why the patients needed to remain in the ICU other than the presence of chest tubes, which may be mandated by policy, rather than hemodynamic need. Therefore, hospital LOS rather than ICU-LOS may have been a more accurate reflection of the intended variable of interest. Nonetheless, ICU-LOS is merely a surrogate for underlying issues (ie, chylothorax), and it is those problems that require clinical focus and, if possible, modification to affect outcome.

Regardless of the limitations, clinically important questions remain. (1) What factors are related to short-term outcomes and thus potentially to long-term outcomes? (2) Which of these factors are modifiable? The answers may lie in surgical innovation. As we moved from classic atriopulmonary to lateral tunnel and extracardiac Fontan, staged palliation and surgical advancement were central drivers of improved outcomes. ${ }^{1}$ What is the next phase of surgical improvement? This study as well as the literature suggest CPB time is associated with ICU-LOS. ${ }^{1,3,5}$ It is also one of the few preoperative (previous bidirectional Glenn [BDG] performed with $\mathrm{CPB}$ ) and intraoperative factors that is directly modifiable. As such, the merits of performing the BDG and extracardiac Fontan anastomoses without $\mathrm{CPB}$ should be considered. CPB and the ensuing cascade of inflammatory cytokines and fluid shifts are associated with worse immediate outcomes, including increased bleeding, medium-term outcomes, 
such as low cardiac output syndrome, fluid overload, and AKI, and long-term outcomes, including LOS and neurodevelopmental outcomes. At centers using this "off-pump" innovation, LOS post-BDG and post-Fontan are shorter than the national aggregate. ${ }^{9}$

Postoperative management factors that this study does not address but are important include duration of mechanical ventilation, early chest tube removal, AKI, and fluid overload. If a patient is not extubated in the operating room (OR), this can prolong ICU-LOS. ${ }^{10}$ Another factor that plays a role in overall Fontan LOS is chest tube output and removal criteria. In this study, routine fenestration was not used; however, Lemler and colleagues ${ }^{11}$ demonstrated that nonfenestration is associated with prolonged chest tube output and hospital LOS. Both OR extubation and early chest tube removal have the additional benefit of allowing early mobilization and cardiac rehabilitation, which may decrease postoperative complications and LOS. ${ }^{12}$ Finally, AKI and fluid overload, common after the Fontan operation, have an association with longer LOS and should be proactively prevented (ie, avoidance of $\mathrm{CPB}$ ) or, if not, identified and treated. ${ }^{13,14}$

Surgical innovation to improve both short- and long-term outcomes after Fontan repair is possible. Avoiding CPB for BDG and Fontan anastomoses addresses CPB as a contributor to poor outcomes. OR extubation, strategies to promote early chest tube removal, and prevention or avoidance of AKI and fluid overload may also improve LOS and ultimately long-term Fontan outcomes.

\section{References}

1. Rogers LS, Glatz AC, Ravishankar C, Spray TL, Nicolson SC, Rychik J, et al. 18 years of the Fontan operation at a single institution: results from 771 consecutive patients. J Am Coll Cardiol. 2012;60:1018-25.
2. Salvin JW, Scheurer MA, Laussen PC, Mayer JE Jr, Del Nido PJ, Pigula FA, et al. Factors associated with prolonged recovery after the Fontan operation. Circulation. 2008;118:S171-6.

3. Downing TE, Allen KY, Glatz AC, Rogers LS, Ravishankar C, Rychik J, et al. Long-term survival after the Fontan operation: twenty years of experience at a single center. J Thorac Cardiovasc Surg. 2017; 154:243-53.e2.

4. Downing TE, Allen KY, Goldberg DJ, Rogers LS, Ravishankar C, Rychik J, et al. Surgical and catheter-based reinterventions are common in long-term survivors of the Fontan operation. Circ Cardiovasc Interv. 2017;10(9).

5. Ono M, Burri M, Balling G, Beran E, Cleuziou J, Pabst von Ohain J, et al. Predicted clinical factors associated with the intensive care unit length of stay following total cavopulmonary connection. J Thorac Cardiovasc Surg. 2019; 157:2005-13.e3.

6. Ohuchi H, Miyazaki A, Negishi J, Hayama Y, Nakai M, Nishimura K, et al. Hemodynamic determinants of mortality after Fontan operation. Am Heart J. 2017; 189:9-18.

7. Cooper DS, Kwiatkowski DM, Goldstein SL, Krawczeski CD. Acute kidney injury and cardiorenal syndromes in pediatric cardiac intensive care. Pediatr Crit Care Med. 2016;17(8 suppl 1):S250-6.

8. Mah KE, Hao S, Sutherland SM, Kwiatkowski DM, Axelrod DM, Almond CS, et al. Fluid overload independent of acute kidney injury predicts poor outcomes in neonates following congenital heart surgery. Pediatr Nephrol. 2018;33: 511-20.

9. Mainwaring RD, Reddy VM, Hanley FL. Completion of the three-stage Fontan pathway without cardiopulmonary bypass. World J Pediatr Congenit Heart Surg. 2014;5:427-33.

10. Morales DLS, Carberry K, Heinle JS, McKenzie ED, Fraser CD Jr, Diaz LK. Extubation in the operating room after Fontan's procedure: effect on practice and outcomes. Ann Thorac Surg. 2008;86:576-82.

11. Lemler MS, Scott WA, Leonard SR, Stromberg D, Ramaciotti C. Fenestration improves clinical outcome of the Fontan procedure: a prospective, randomized study. Circulation. 2002;105:207-12.

12. Sunstrom RE, Muralidaran A, Gerrah R, Reed RD, Good MK, Armsby LR, et al. A defined management strategy improves early outcomes after the Fontan procedure: the PORTLAND protocol. Ann Thorac Surg. 2015;99: $148-55$.

13. Esch JJ, Salvin JM, Thiagarajan RR, Del Nido PJ, Rajagopal SK. Acute kidney injury after Fontan completion: risk factors and outcomes. J Thorac Cardiovasc Surg. 2015;150:190-7.

14. Algaze CA, Koth AM, Faberowski LW, Hanley FL, Krawczeski CD, Axelrod DM. Acute kidney injury in patients undergoing the extracardiac Fontan operation with and without the use of cardiopulmonary bypass. Pediatr Crit Care Med. 2017; 18:34-43. 